\title{
Past experience, recency, and spontaneous recovery in choice behavior
}

\author{
JAMES E. MAZUR \\ Southern Connecticut State University, New Haven, Connecticut
}

\begin{abstract}
Pigeons' responses on two keys were recorded before and after the percentage of reinforcers delivered by each key was changed. In each condition of Experiment 1, the reinforcement percentage for one key was $50 \%$ for several sessions, then either $70 \%$ or $90 \%$ for one, two, or three sessions, and then $50 \%$ for another few sessions. At the start of the second and third sessions after a change in reinforcement percentages, choice percentages often exhibited spontaneous recovery-a reversion to the response percentages of earlier sessions. The spontaneous recovery consisted of a shift toward a more extreme response percentage in some cases and toward a less extreme response percentage in other cases, depending on what reinforcement percentages were previously in effect. In Experiment 2 , some conditions included a 3-day rest period before a change in reinforcement percentages, and other conditions included no such rest days. Slightly less spontaneous recovery was observed in conditions with the rest periods, suggesting that the influence of prior sessions diminished with the passage of time. The results are consistent with the view that choice behavior at the start of a new session is based on a weighted average of the events of the past several sessions.
\end{abstract}

In the past, nearly all experiments on choice using operant conditioning procedures reported only "steadystate" results - the choice responses of subjects whose behavior appeared to be relatively stable because they had been exposed to the same reinforcement contingencies for many sessions. In recent years, however, there has been increasing interest in choice behavior during periods of transition-periods when the reinforcement contingencies have just changed, and the subject's behavior is in the process of adapting to the new contingencies. These experiments have used a variety of different species and experimental procedures (e.g., Couvillon \& Bitterman, 1985, 1988, 1991; Dreyfus, 1991; Kacelnik, Krebs, \& Ens, 1987; Mark \& Gallistel, 1994), and they have uncovered a number of valuable findings about choice behavior in transition.

My colleagues and I have used a procedure in which it is possible to obtain many acquisition curves from each subject (Bailey \& Mazur, 1990; Mazur, 1992; Mazur \& Ratti, 1991). Each condition begins with a period with equal reinforcement contingencies on two response keys, and then the development of preference is observed when one key suddenly begins to deliver more reinforcers than the other. Previous studies with this procedure examined how rates of transition were affected by a number of different factors, such as the overall probability of rein-

This research was supported by Grant MH 38357 from the National Institute of Mental Health. The author thanks Jeffrey Colafrancesco, Peter Kelly, and Margaret Nygren for their help in different phases of the research. Correspondence should be addressed to J. E. Mazur, Psychology Department, Southern Connecticut State University, New Haven, CT 06515 (e-mail: mazur@scsu.ctstateu.edu). forcement, the size of a change in probabilities, and the overall rate of reinforcement.

The results of these experiments contradicted the predictions of several models of transitional choice behavior, including Myerson's kinetic model (Myerson \& Miezin, 1980; see also Myerson \& Hale, 1988), Staddon's ratioinvariance model (Staddon, 1988; Staddon \& Horner, 1989), and the linear-operator model (Bush \& Mosteller, 1955). However, the results could be described quite well by a simple mathematical model similar to one proposed by Couvillon and Bitterman (1985). The model states that each response alternative, $i$, has a separate strength, $V_{i}$. Response strength increases each time the response is reinforced and decreases each time the response is not reinforced. Each time a response is reinforced, $V_{i}$ increases as follows:

$$
\Delta V_{i}=r(1-V i) \text {, }
$$

where $\Delta V_{i}$ is the change in strength of $V_{i}$, and $r$ is a reinforcement parameter that can range from 0 to 1 . After each response that is not reinforced, the strength of the response decreases as follows:

$$
\Delta V_{i}=n\left(-V_{i}\right),
$$

where $n$ is a nonreinforcement parameter that can range from 0 to 1 . A simple matching rule is used to translate from the independent strengths of two responses $\left(V_{i}\right.$ and $V_{2}$ ) to the probability that one will occur:

$$
p_{1}=\frac{V_{1}}{V_{1}+V_{2}}
$$

Although the results of several experiments were well described by this model, it is too simple to accommodate 
some of the complexities of behavior observed in simple choice situations. For example, Mazur (1995) found a type of spontaneous recovery that was not predicted by this model (nor by most other models of transitional choice behavior, including all of those mentioned so far). The spontaneous recovery took the following form: After a few sessions in which each of two response keys delivered about $50 \%$ of the reinforcers, the reinforcement percentage for one key (the "rich key") switched to $90 \%$. Responding on the rich key rapidly increased, and, by the end of one session, subjects were making about $75 \%$ of their responses on this key. However, at the beginning of the next session, the percentage of responses on the rich key dropped to about $60 \%$. This phenomenon was called spontaneous recovery because it consisted of a reversion to a previously reinforced choice proportion. Responding on the rich key increased during the course of the second session, but similar spontaneous recovery was observed at the beginnings of the next few sessions (though it became progressively smaller over sessions).

The reason most models of transitional choice behavior cannot account for this spontaneous recovery is straightforward: They do not treat the beginning of a new session in any special way, and they assume that behavior at the start of a session will simply resume where it left off at the end of the previous session. Mazur (1995) proposed that this assumption is not defensible and that behavior at the start of a new session reflects some sort of weighted average of the events of the past several sessions. To model this spontaneous recovery, Mazur ran a series of computer simulations based on Equations 1-3, but with one change. At the start of each simulated session, the values of $V_{1}$ and $V_{2}$ were not set at the final values from the previous session but were set at a weighted average of their values from the previous five sessions. Values of $V_{1}$ and $V_{2}$ from the most recent session were given the greatest weight, and values from the fifth most recent session were given the least weight. The magnitude and time course of the spontaneous recovery in the computer simulations corresponded closely to those in the actual data.

Similar ideas about spontaneous recovery and the effects of previous experiences have been proposed by others. Mazur (1995) noted that Estes's (1955) model of stimulus fluctuations, which was designed to account for the spontaneous recovery of a single response, can be easily extended to predict spontaneous recovery in choice situations. This approach is also similar to Spear's (1967, 1971) hypothesis that spontaneous recovery is a type of proactive interference, in which older learning interferes with more recent learning. More recently, J. A. Devenport and L. D. Devenport (1993; L. D. Devenport \& J. A. Devenport, 1994) proposed a model of how past experiences combine to affect current choices in foraging situations. This model is more specific than Mazur's analysis of spontaneous recovery because it makes recommendations about exactly what weights should be assigned to past experiences. The model states that the influence of every past experience on an organism's current choice behavior is an inverse function of its recency.
For now, the details of these models are less important than the general hypothesis that choice behavior at the start of a new session depends on some type of weighted average of the subject's experiences over the past several sessions. It follows from this hypothesis that a subject's choice behavior at the end of one previous session will often be a poor predictor of behavior at the start of the next session. For instance, imagine that for one subject, the left-key reinforcement percentages in the last four sessions have been $50 \%, 50 \%, 50 \%$, and $90 \%$. For a second subject, the left-key reinforcement percentages have been $50 \%, 90 \%, 90 \%$, and $90 \%$. At the end of the fourth session, these subjects might exhibit similar preference for the left key. However, at the start of the next session, the first subject should exhibit less preference for the left key (and more spontaneous recovery in the direction of indifference) because this subject has had fewer sessions with the $90 \%$ reinforcement proportion.

The present experiments were conducted to obtain additional information on how the events of past sessions combine to affect choice behavior in subsequent sessions. One purpose of Experiment 1 was to demonstrate that spontaneous recovery in choice behavior can appear either as a shift toward indifference or as a shift toward more extreme preference for one alternative (depending on the reinforcement percentages used). Experiment 2 was designed to determine if the influence of past sessions, and the amount of spontaneous recovery, can be altered by the simple passage of time. That is, will the experiences of previous sessions have less effect on current choice behavior if several days have passed since those sessions occurred, even if the subject receives no additional exposure to the choice situation in the intervening days?

\section{EXPERIMENT 1}

In Mazur's (1995) experiments, the clearest examples of spontaneous recovery all consisted of a shift toward a less extreme choice proportion, and it could be argued that they were at least partly caused by a tendency to begin each session closer to indifference. But if these shifts at the beginning of a session actually reflected the reinforcement contingencies of previous sessions, with the appropriate experimental design it should be possible to observe cases of spontaneous recovery that consist of a shift toward a more extreme choice proportion (i.e., a shift away from $50 \%$ responding on each key). Experiment 1 was designed to look for this type of spontaneous recovery. This experiment was also designed to determine whether the degree of preference for one alternative and the amount of spontaneous recovery will depend on the number of sessions in which that alternative had a higher reinforcement percentage.

\section{Method}

Subjects. The subjects were 5 of the 6 White Carneaux pigeons that had previously served in the experiments of Mazur (1995) and in other experiments with similar procedures. (The 6th pigeon died 
near the beginning of the present experiment.) The subjects received most of their food in the experimental chambers but were given additional food after each session, when necessary, to keep them at approximately $80 \%$ of their free-feeding weights.

Apparatus. The experimental chamber was $30 \mathrm{~cm}$ long, $30 \mathrm{~cm}$ wide, and $30.5 \mathrm{~cm}$ high. Recessed in the right sidewall of the chamber, $22.8 \mathrm{~cm}$ above the floor, were three response keys, separated by $5.6 \mathrm{~cm}$, and each measuring $2.5 \mathrm{~cm}$ in diameter. The center key was not used in this experiment. Each of the two outside keys could be transilluminated by red or green light produced by two 2-W bulbs mounted behind the keys. A force of about $0.1 \mathrm{~N}$ was required to operate each key. A hopper that could present grain for a specified interval was situated $12 \mathrm{~cm}$ below the center key. When the grain was presented, the hopper was illuminated with white light from a $2-W$ bulb mounted above it. Two $2-W$ houselights were mounted above the Plexiglas ceiling of the chamber.

The chamber was enclosed in a sound-attenuating wooden box that had an exhaust fan for ventilation and for masking extraneous noises. An IBM-compatible personal computer was programmed in the Medstate language to control all stimuli and to record the responses.

Procedure. All pigeons had considerable experience with the procedure through their participation in previous experiments, so no additional training was needed at the start of this experiment The complete experiment included 24 conditions with usable data plus a few conditions in which equipment failures or experimenter errors made the data unusable. Conditions with unusable data were later repeated to obtain an equal number of replications with the appropriate parameters for all subjects. For ease of discussion, each condition will be described as having three separate phases: a baseline phase, a transition phase, and a recovery phase. However, the phases of adjacent conditions overlapped, because the recovery phase of one condition also served as the baseline phase for the subsequent condition. Baseline and recovery phases included four, five, or six sessions in which the scheduled reinforcement percentages were $50 \%$ for each key. The number of baseline sessions was varied unpredictably so that this number could not serve as a cue for when the transition phase would begin. Transition phases included one, two, or three sessions in which the reinforcement percentage was higher for one key than for the other. For each condition, the key with the higher reinforcement percentage in the transition phase will be called the rich key, even though reinforcement percentages were the same for the two keys in the baseline and recovery phases.

All sessions lasted for $30 \mathrm{~min}$ and were usually conducted 6 days a week. At the beginning of a session, the houselights came on and the two response keys were illuminated-the left key red and the right key green. The houselights remained on for the entire session, except during reinforcement periods. Every effective peck on either key produced a $0.1-\mathrm{sec}$ darkening of both keys as feedback. If a response resulted in reinforcement, the houselights and keylights were turned off and the hopper was illuminated and raised to present grain for $2.5 \mathrm{sec}$. After reinforcement, the houselights and keylights were again illuminated. At the end of a session, all chamber lights were turned off. This procedure was used throughout the experiment, and the only differences among sessions were the reinforcement percentages for the two keys, as described below.

In all sessions, reinforcers were assigned by a single variableinterval (VI) 30-sec schedule: Once per second, if no reinforcer was currently assigned to one of the two keys, there was a .0333 probability that a reinforcer would be assigned to one of the two keys. Assignment to the left or right key was determined by a pseudorandom sequence that ensured that the actual percentage of reinforcers delivered by each key was close to the scheduled percentage. Once a reinforcer was assigned to a key, no further reinforcers were assigned to either key until that reinforcer was delivered. The procedure also included a 2 -sec changeover delay (COD)-that is, $2 \mathrm{sec}$ had to elapse after each changeover response before a response could be reinforced. If a reinforcer was assigned to a key, it was delivered following the first post-COD response on that key.

The two independent variables in this experiment were the number of transition sessions in a condition and the reinforcement percentages for the rich key during the transition sessions. The experiment used a $3 \times 2$ design: The number of transition sessions

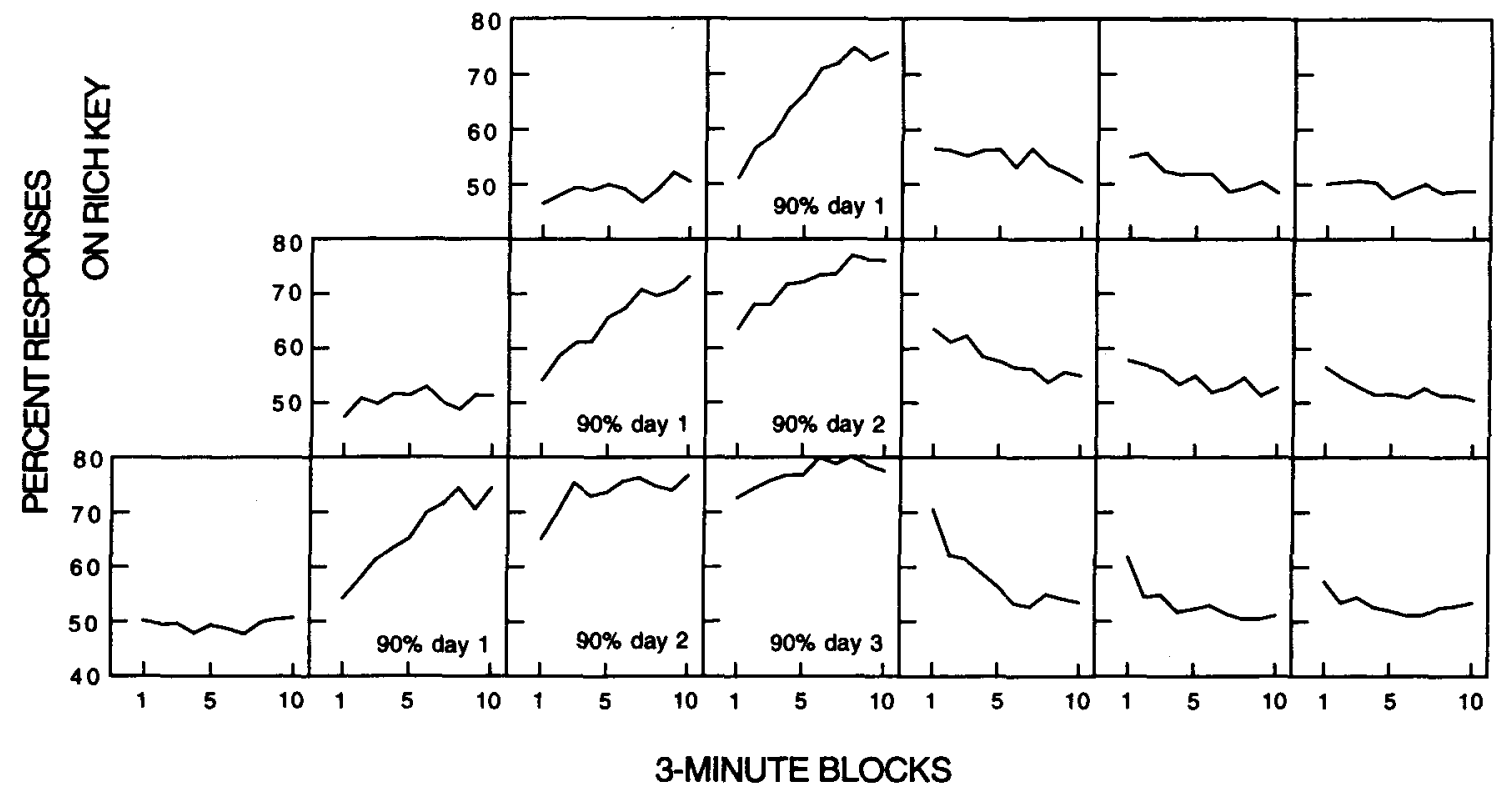

Figure 1. The three rows show the results from the three different conditions of Experiment 1 in which the rich key delivered $90 \%$ of the reinforcers on transition days. Rich-key response percentages, averaged across subjects and replications, are shown in 3-min blocks for the last baseline session, all transition sessions, and the first three recovery sessions. The reinforcement percentage for the rich key is labeled for every transition session; the percentage for all unlabeled panels was $50 \%$. 
was one, two, or three, and the rich-key reinforcement probability in these sessions was either $70 \%$ or $90 \%$. For each subject, each of the six combinations of these two variables was repeated in 4 different conditions, for a total of 24 conditions. The order of conditions was counterbalanced across subjects. The rich key was the left key in half of the conditions and the right key in the other half.

\section{Results}

The three rows in Figure 1 show the results from the three different conditions in which the rich key delivered $90 \%$ of the reinforcers on transition days. The results are averaged across the 5 subjects and six replications of each condition, so every panel in Figure 1 is an average of 30 sessions. This figure shows the percentage of responses on the rich key in 3-min blocks, for the last baseline session, all transition sessions, and the first three recovery sessions. The reinforcement percentage for the rich key is labeled for every transition session; the percentage for all unlabeled panels was $50 \%$. Figure 2 uses the same format to present the results from conditions with a richkey reinforcement percentage of $70 \%$ on transition days.

The first panel in every row of Figures 1 and 2 represents the last baseline session of each condition. In all cases, the percentage of responses on the rich key was approximately $50 \%$. In the one or more transition sessions that followed, responding on the rich key increased, eventually reaching a level of about $75 \%$ in Figure 1 and slightly over $60 \%$ in Figure 2 . Response percentages in the transition sessions never reached the point of matching, since the corresponding reinforcement percentages were $90 \%$ and $70 \%$. In the conditions with more than one transition session (the two bottom rows in each figure), spontaneous recovery was exhibited on Transition Days 2 and 3 . That is, at the beginning of these sessions, response percentages were lower than at the end of the previous session.

To assess the reliability of this evidence for spontaneous recovery, the following analysis was done. For each subject and each of the six cases where spontaneous recovery might be seen (i.e., the six panels labeled "day 2" or "day 3" in Figures 1 and 2), the mean rich-key response percentage from the first 3-min block was compared with the mean response percentage from the last 3-min block of the preceding session. A repeated measures analysis of variance (ANOVA) found that the response percentages were significantly lower in the first 3-min blocks of the succeeding sessions $[F(1,4)=35.55, p<.01]$.

By the end of the last transition session, response percentages were similar for conditions with the same reinforcement percentage, regardless of whether this was the first, second, or third transition session. For the $90 \%$ reinforcement conditions, the response percentages in the last 3-min blocks of the last transition session were $74 \%$, $76 \%$, and $77 \%$, for the conditions with one, two, and three transition sessions, respectively. The corresponding response percentages for the three conditions with $70 \%$ reinforcement were $63 \%, 63 \%$, and $59 \%$, respectively. A repeated measures ANOVA was conducted using the response percentages from these final 3-min blocks of the last transition session. The ANOVA found a significant effect of transition reinforcement percentage $[F(1,4)=$ $96.62, p<.001]$ but no significant effect of the number of transition sessions $[F(2,8)=0.53]$. The interaction of these two factors was not significant $[F(2,8)=3.10]$.

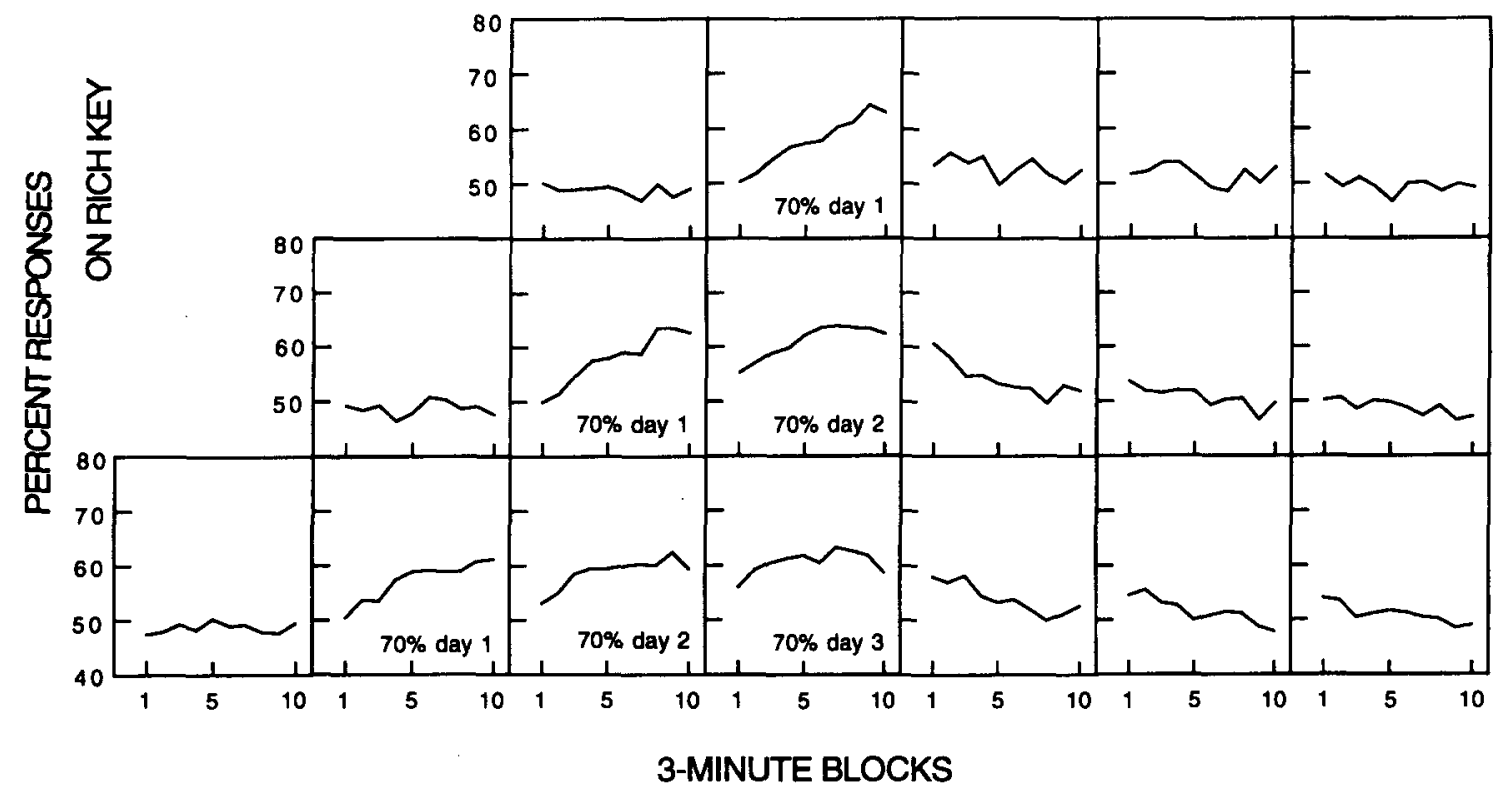

Figure 2. The three rows show the results from the three different conditions of Experiment 1 in which the rich key delivered $70 \%$ of the reinforcers on transition days. Rich-key response percentages, averaged across subjects and replications, are shown in 3-min blocks for the last baseline session, all transition sessions, and the first three recovery sessions. The reinforcement percentage for the rich key is labeled for every transition session; the percentage for all unlabeled panels was $50 \%$. 
Although response percentages at the end of the final transition sessions were similar for all conditions with the same reinforcement percentage, behavior in the subsequent recovery sessions was not. In general, the results from the recovery sessions (the last three panels in each row of Figures 1 and 2) were consistent with the prediction that choice behavior would depend on both the number of transition sessions and the reinforcement percentage during those transition sessions. At the beginning of the first recovery session of a condition, rich-key response percentages were higher after $90 \%$ transition sessions than after $70 \%$ sessions, and they were also usually higher when the condition included more transition sessions. A repeated measures ANOVA was conducted using the response percentages from the first 3-min blocks of the first recovery session of each condition. The ANOVA found a significant effect of transition reinforcement percentage $[F(1,4)=8.63, p<$ $.05]$ and a significant effect of the number of transition sessions $[F(2,8)=9.18, p<.01]$. The interaction of these two factors was also significant $[F(2,8)=10.52, p<.01]$.

If spontaneous recovery was present in the second and third recovery sessions (last two columns in Figures 1 and 2), it would appear as a higher rich-key response percentage at the start of a session than at the end of the preceding session. As expected, the amount of spontaneous recovery depended on the number of transition sessions and on the reinforcement percentages used in those transition sessions. For the conditions with 2 or 3 days of $90 \%$ reinforcement (bottom two rows of Figure 1), there was evidence for spontaneous recovery on both the 2 nd and 3 rd recovery days. For the condition with only 1 day of $90 \%$ reinforcement, there was evidence for spontaneous recovery on the 2 nd recovery day but not on the $3 \mathrm{rd}$. For the conditions with $70 \%$ reinforcement, Figure 2 shows less spontaneous recovery. The condition with 3 days of $70 \%$ reinforcement (bottom row) showed some spontaneous recovery on both the 2 nd and 3rd recovery days, but there was little or no sign of spontaneous recovery in the conditions with 1 or 2 days of $70 \%$ reinforcement.

The overall reliability of this spontaneous recovery was assessed with the same type of analysis used for the spontaneous recovery in the transition sessions. In this analysis, rich-key response percentages in the first 3-min block of the second recovery session were compared with those in the last 3-min block of the first recovery session. An ANOVA found significantly higher response percentages at the start of the second session than at the end of the first session $[F(1,4)=44.21, p<.01]$. A similar analysis found higher rich-key response percentages at the start of the third recovery session than at the end of the previous session $[F(1,4)=16.73, p<.05]$. In summary, the evidence for spontaneous recovery toward a more extreme response percentage was statistically significant in both the second and third recovery sessions, although it was not observed in every case shown in Figures 1 and 2.

\section{Discussion}

The results of Experiment 1 clearly demonstrate that choice behavior at the start of a new session cannot be predicted solely on the basis of the subject's behavior at the end of the previous session. When the reinforcement percentages had recently changed, choice behavior at the start of a new session exhibited spontaneous recoverya shift toward the behavior of previous sessions, when a different reinforcement percentage was in effect. This spontaneous recovery was seen both as a shift toward indifference (during the transition sessions) and as a shift toward a more extreme preference for the previously rich key (during the recovery sessions).

During the recovery sessions, the degree of preference for the previously rich key depended on the number of transition sessions and on whether the rich-key reinforcement percentage had been $70 \%$ or $90 \%$. The top row of Figure 2 shows that when there was only one transition session with a reinforcement percentage of $70 \%$, this experience had little lasting effect on choice behavior, even in the first recovery session. At the other extreme, in the condition with three transition sessions and a reinforcement percentage of $90 \%$ (bottom row of Figure 1), rich-key response percentages were high at the beginning of all three recovery sessions that followed. In the conditions between these two extremes, recovery sessions showed intermediate levels of preference for the rich key and intermediate amounts of spontaneous recovery.

All of these results are consistent with the hypothesis that choice behavior at the start of a new session is based on a weighted average of the events of the past several sessions. The following analysis shows that the results can be well approximated with computer simulations using Equations 1-3 and a rule that states that the values of $V_{1}$ and $V_{2}$ at the start of a session are weighted averages of their values from previous sessions.

The computer simulations were similar to those used by Mazur $(1992,1995)$ for previous experiments. The parameters $r$ and $n$ in Equations 1 and 2 were both set at .002 . A constant response rate of one response per second was used, which roughly approximated the average response rate in the experiment. The COD was simulated by not reinforcing either of the first two responses on one key after a changeover from the other key. The computer program conducted a series of simulations, each consisting of the appropriate number of baseline, transition, and recovery sessions. Simulated responses were chosen on a probabilistic basis according to Equation 3, using the current values of $V_{1}$ and $V_{2}$. Because responses and reinforcers were chosen probabilistically, individual simulations showed considerable variability. To reduce the variability and determine the average results predicted by the model, 100 simulations were conducted for each of the six conditions of Experiment 1.

To account for the effects of previous sessions, the values of $V_{1}$ and $V_{2}$ at the start of each session were set at weighted averages of the final values from the previous five sessions. Many different methods for selecting these weights could be tried; however, for the present experiment, the weights were based on the model of J. A. Devenport and L. D. Devenport (1993; L. D. Devenport \& J. A. Devenport, 1994). This model proposes that the 


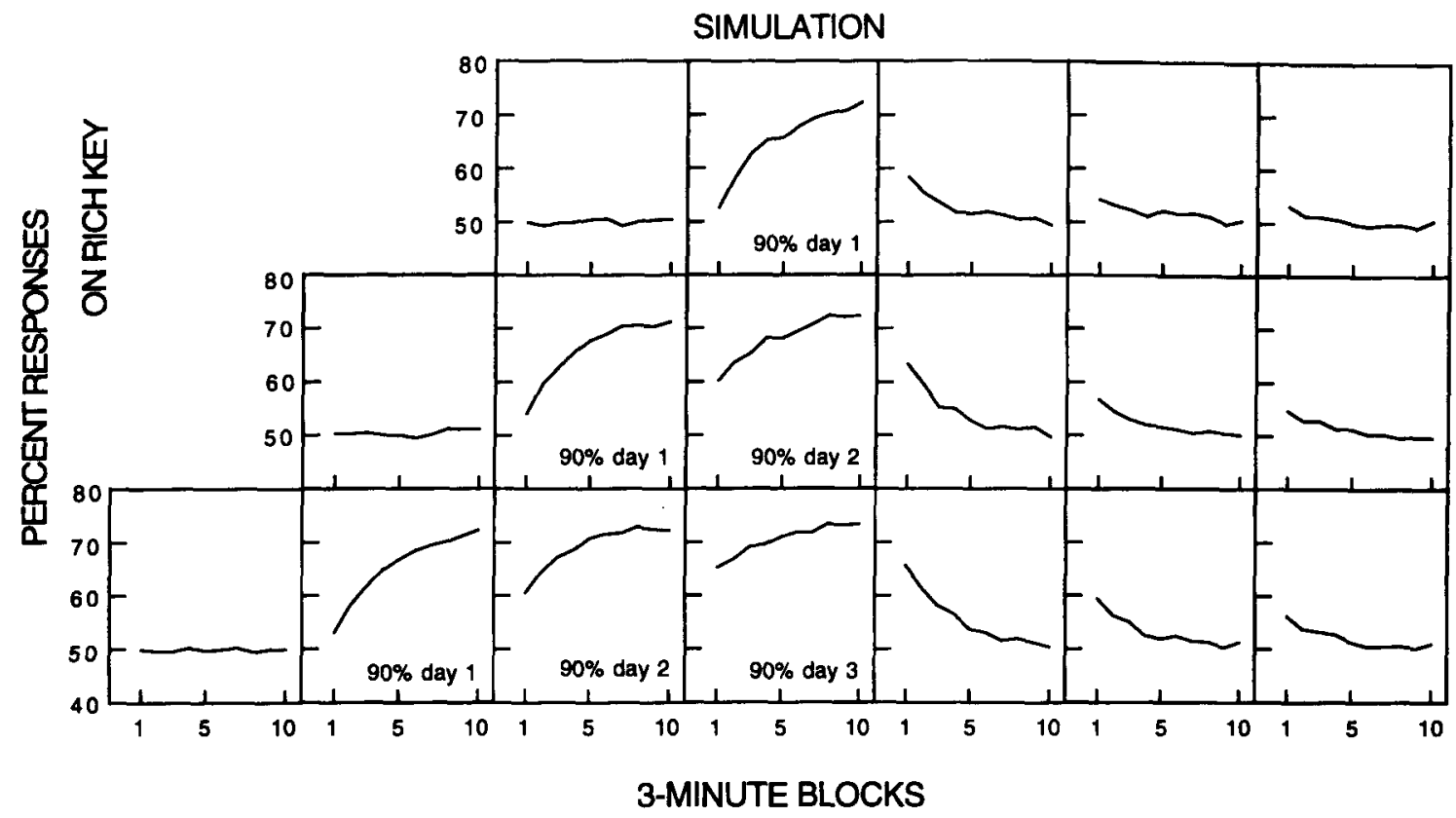

Figure 3. The results of computer simulations based on Equations 1-4 for the conditions in Experiment 1 in which the rich key delivered $90 \%$ of the reinforcers. The simulations are presented in the same form as the actual results shown in Figure 1.

weight for each past experience should be inversely related to its recency, as follows:

$$
W_{x}=\frac{1 / t_{x}}{\sum_{k=1}^{n} 1 / t_{k}}
$$

$W_{x}$ is the weight assigned to a particular experience, $t_{x}$ is the time since that experience took place, and $n$ is the number of experiences being given a weight. The numerator of this expression is simply the inverse of $t_{x}$, and the denominator is included so that the sum of all $n$ weights equals 1 . This model contains no free parameters, but it was able to predict the results of several studies on patch selection with dogs, chipmunks, and ground squirrels.

For these simulations, only the past five sessions were given a weight, and Equation 4 yields weights for these five sessions of $.438, .219, .146, .110$, and .088 . These weights were multiplied by the values of $V_{1}$ and $V_{2}$ to obtain the values for the start of each new session.

Figures 3 and 4 present the results from these computer simulations in the same form as Figures 1 and 2. The simulations captured the main features of the actual data. The simulations showed both types of spontaneous recovery - toward indifference in the 2 nd and 3rd transition days, and toward a higher response percentage in the 2nd and 3 rd recovery days. In the first recovery sessions, preference for the previously rich key was greater after $90 \%$ reinforcement than after $70 \%$ reinforcement, and it was greater with more transition sessions. Similarly, the magnitude of the spontaneous recovery in Recovery Days 2 and 3 were greater with the higher reinforcement percentage and with more transition sessions.
Although these simulations were able to account for the main trends in the data, they should not be viewed as strong evidence for the particular model described by Equations 1-3, nor for the particular weights derived from Equation 4. Similar patterns of spontaneous recovery were obtained with simulations using fairly different sets of weights. For example, when the weights given to previous sessions decreased linearly, and even when equal weights were asssigned to the previous five sessions, the resultant simulations showed patterns similar to those in Figure 4, though the magnitude of the spontaneous recovery differed slightly (cf. Mazur, 1995). The purpose of these computer simulations was merely to demonstrate that the patterns of acquisition, reversal, and spontaneous recovery observed in this experiment are consistent with the general hypothesis that the events of previous sessions have independent effects on choice behavior. As discussed in more detail elsewhere (L. D. Devenport \& J. A. Devenport, 1994; Mazur, 1995), models that do not assign separate and independent weights to the events of previous sessions cannot account for this type of spontaneous recovery. The common practice in models of acquisition of simply updating a single aggregate measure for the strength of each response alternative (such as single values of $V_{1}$ and $V_{2}$ in Equations 1-3 in their simplest forms) cannot explain the discontinuities that are observed from the end of one session to the beginning of the next.

\section{EXPERIMENT 2}

Equation 4, which L. D. Devenport and J. A. Devenport (1994) called a temporal weighting rule, suggests 


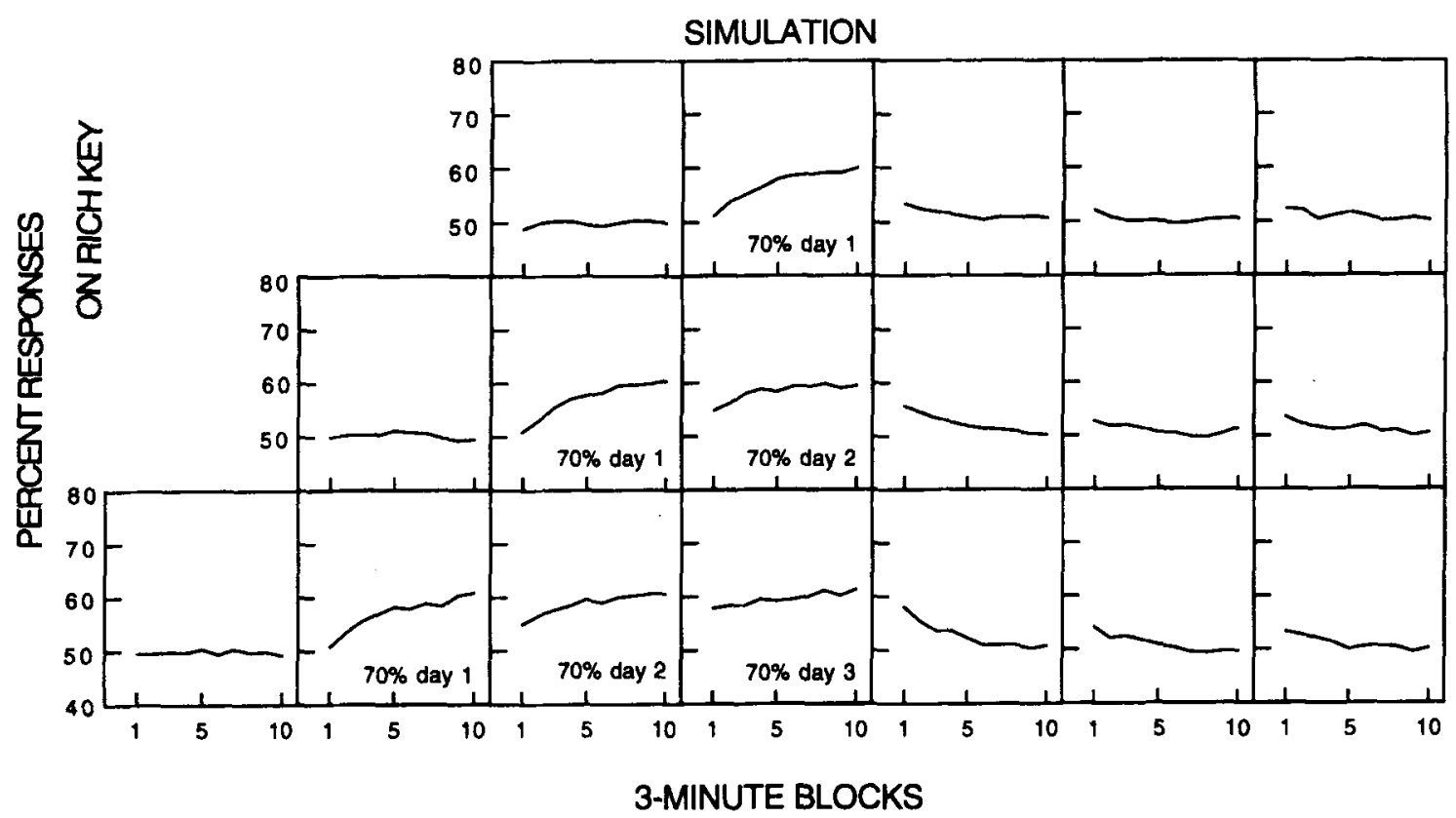

Figure 4. The results of computer simulations based on Equations 1-4 for the conditions in Experiment 1 in which the rich key delivered $70 \%$ of the reinforcers. The simulations are presented in the same form as the actual results shown in Figure 2.

that the influence of a past experience on current choice behavior decreases with the passage of time, regardless of what events fill the intervening time. If this rule is correct, it should be possible to alter the amount of spontaneous recovery observed in a choice situation by inserting 1 or more rest days between sessions. In some conditions of Experiment 2, subjects received several sessions with both keys delivering $50 \%$ of the reinforcers, followed immediately by several sessions with one key delivering either $70 \%$ or $90 \%$ of the reinforcers. In other conditions, subjects received a 3-day rest between the $50 \%$ phase and the $70 \%$ (or $90 \%$ ) phase. If the influence of past sessions decreases simply with the passage of time, there should be less spontaneous recovery after the 3-day rest, because the $50 \%$ sessions will be farther in time from the $70 \%$ (or $90 \%$ ) sessions.

\section{Method}

Subjects and Apparatus. The subjects were the same as those in Experiment 1, and the same experimental chamber was used.

Procedure. The procedure followed for the daily 30 -min sessions was identical to that of Experiment 1, with a single VI 30-sec timer assigning reinforcers to the two response keys. The experiment included 16 conditions with usable data, plus a few conditions in which equipment failures or experimenter errors made the data unusable. Conditions with unusable data were later repeated to obtain an equal number of replications with the appropriate parameters for all subjects. Every condition began with a baseline phase and ended with a transition phase. Each baseline phase consisted of four, five, or six sessions with each key delivering approximately $50 \%$ of the reinforcers. Each transition phase consisted of three sessions. In the transition sessions, the rich-key reinforcement percentage was $70 \%$ for eight conditions and $90 \%$ for the other eight conditions.
Four of the conditions with each reinforcement percentage were rest conditions: The subjects received 3 rest days between the end of the baseline phase and the beginning of the transition phase. On rest days, the subjects were not placed in the experimental chamber, but they were given enough food in their home cages to maintain their body weights. The other four conditions with each reinforcement percentage were no-rest conditions: There were no rest days between the baseline phase and the transition phase. However, so that rest days could not serve as discriminative stimuli signaling the transition from $50 \%$ to some other reinforcement percentage, rest days were also included at other times during the course of the experiment. Most of the no-rest conditions had 1,2, or 3 rest days inserted somewhere in the middle of the baseline phase. For example, the sequence for one no-rest condition was 2 baseline sessions, 3 rest days, 4 more baseline sessions, and 3 transition sessions. The placement of rest days was restricted, however, so that at least 2 consecutive baseline sessions immediately preceded the transition phase in all no-rest conditions. In addition, a single rest day sometimes separated the end of one condition from the beginning of the next. Because of the presence of these additional rest days, it was not possible to predict when rest days would occur or what reinforcement percentage would follow them.

\section{Results}

Figure 5 shows the results from the conditions with $90 \%$ rich-key reinforcement (top row) and $70 \%$ rich-key reinforcement (bottom row). The results are averaged across the 5 subjects and the four replications of each condition, so every panel is an average of 20 sessions. Figure 5 shows the percentage of responses on the rich key in 3-min blocks, for the last baseline session and the first two transition sessions of each condition. The results are shown for conditions with a 3-day rest after the last baseline session (dashed lines) and for conditions with no rest days (solid lines). The first panel in each row 


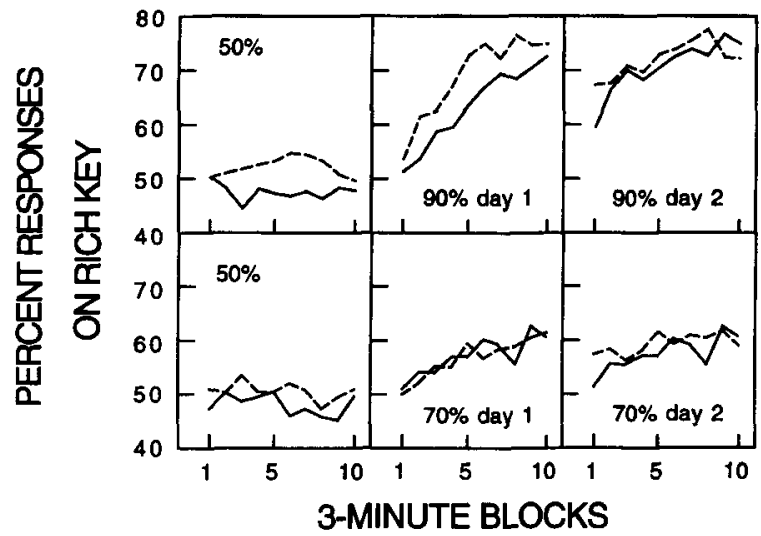

Figure 5. Rich-key response percentages are shown in 3-min blocks for the last baseline session and the first two transition sessions of each condition of Experiment 2, averaged across subjects and replications. The results are shown for conditions with a 3-day rest after the last baseline session (dashed lines) and for conditions with no rest days (solid lines).

shows that, in the last baseline session, the rich-key response percentages were close to $50 \%$ in all conditions, although they were slightly higher in the conditions with the 3-day rest. The second panels show the development of preference for the rich key on the 1st transition day. With $70 \%$ reinforcement, the increases in preference were similar with and without the 3-day rest. With $90 \%$ reinforcement, rich-key responses appeared to increase more rapidly in conditions with the 3-day rest. However, an ANOVA using response percentages from the entire duration of the 1 st transition day of each condition found no significant effect of the 3-day rest $[F(1,4)=1.50]$. There was a significant effect of reinforcement percentage $[F(1,4)=23.86, p<.01]$, and no significant interaction $[F(1,4)=0.80]$.

If the rest days had an effect on the amount of spontaneous recovery, this effect should be largest at the start of the 2nd transition day, which is the first place that spontaneous recovery should appear. The third panel in each row shows that, for both the $90 \%$ and the $70 \%$ reinforcement conditions, there was slightly less spontaneous recovery at the beginning of these sessions in conditions with the 3-day rest. An ANOVA based on the first 3-min block of each of these sessions found a significant effect of the 3-day rest $[F(1,4)=10.87, p<.05]$. The effect of reinforcement percentage was also significant $[F(1,4)=42.07, p<.01]$, and there was no significant interaction $[F(1,4)=0.45]$.

Although there was a significant difference between the conditions with and without the 3-day rest in the predicted direction, the effect was small. It could be argued that this difference was simply a continuation of the slight difference between the rest and no-rest conditions seen in the baseline sessions. However, several lines of evidence suggest that this was not the case. First, the differences between rest and no-rest conditions were not statistically significant in the last baseline session $[F(1,4)=$
$7.48]$, in the last $3 \mathrm{~min}$ of this session $[F(1,4)=0.71]$, or in the first transition session $[F(1,4)=1.50]$, but they were statistically significant at the start of the second transition session, precisely at the time when the model predicts a difference should appear. Second, the magnitude of the difference between the rest and no-rest conditions was also largest at this time. The difference between rest and no-rest response percentages averaged $3.7 \%$ in the last baseline session (1.6\% in the last $3 \mathrm{~min}$ ) and $2.4 \%$ in the first transition session, but this difference increased to $6.9 \%$ in the first $3 \mathrm{~min}$ of the second transition session. A planned comparison found a significantly greater difference between the rest and no-rest conditions in the first 3-min block of the second transition session than in the last block of the last baseline session and in the first and last blocks of the first transition session $[F(1,4)=8.70, p<.05]$. Third, in the $70 \%$ conditions, response percentages in the rest and no-rest conditions were virtually indistinguishable throughout the first transition session (lower middle panel of Figure 5), yet a difference emerged at the start of the second transition session, as predicted (lower right panel). Thus, although the difference between rest and no-rest conditions was small, it was probably not simply a continuation of the slight differences observed in the baseline sessions.

There was also some indication that the difference between rest and no-rest conditions was larger at the start of the experiment and became smaller at the end of the experiment. Figure 6 shows the results from the first half of the experiment (the first eight conditions), presented in the same form as those in Figure 5. Overall, the results are similar to those from the entire experiment, except that the differences between rest and no-rest conditions were larger on the 2nd transition day (third panel in each row). These results are less reliable because they are based on fewer conditions, but they suggest that the 3-day rest may have had less effect as the experiment progressed.

\section{Discussion}

The results of Experiment 2 support the theory of J. A. Devenport and L. D. Devenport (1993) that the passage of time affects the weighting of past events in their effect on current choice behavior. For this experiment, the theory predicts that, with the 3 -day rest, there should be less spontaneous recovery at the start of the 2 nd transition day. This is because, with the 3-day rest, the most recent session with $50 \%$ reinforcement occurred 5 days earlier, whereas, without the 3-day rest, the most recent $50 \%$ session occurred only 2 days earlier. In this experiment, the effects of the 3-day rest were not large, but the only significant differences between rest and no-rest conditions occurred just where the theory predicted the largest differences - at the start of the second transition session. The small size of the differences between rest and norest conditions is not inconsistent with the predictions of Equations 1-4. Computer simulations for the conditions of this experiment (using the same parameter values as for Experiment 1) produced differences between rest and 


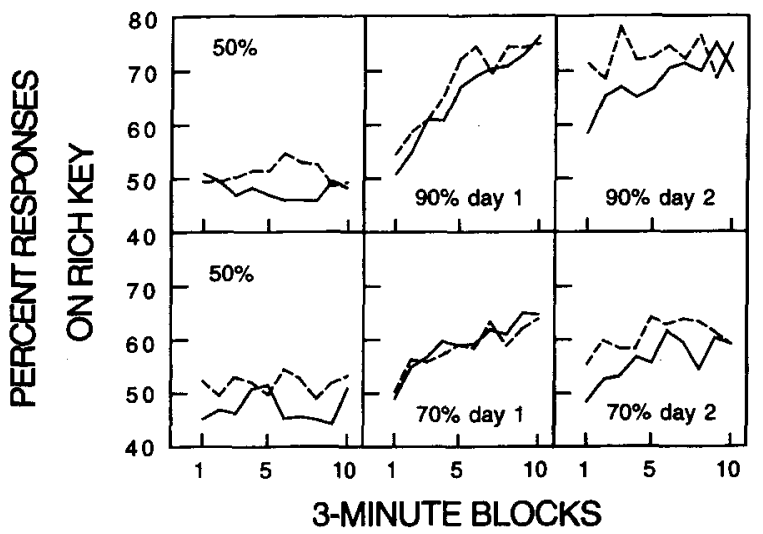

Figure 6. For the first eight conditions of Experiment 2, rich-key response percentages are shown in 3-min blocks for the last baseline session and the first two transition sessions, averaged across subjects and replications. The results are shown for conditions with a 3-day rest after the last baseline session (dashed lines) and for conditions with no rest days (solid lines).

no-rest conditions that were somewhat smaller than those shown in Figure 5. Therefore, as in Experiment 1, the results offer qualitative support for the type of model proposed by J. A. Devenport and L. D. Devenport, but they cannot provide a meaningful test of the specific weights derived from Equation 4.

At first glance, the results of this study may appear to conflict with those of more traditional examples of spontaneous recovery, in which a single response is first reinforced and then extinguished. For example, in the classic experiment of Ellson (1938), a rest period was inserted after the first extinction session, and more spontaneous recovery was observed as the duration of the rest period was increased. In contrast, slightly less spontaneous recovery was observed after a longer rest period in the present experiment. However, there is actually no conflict between the results of these two studies, because, in the present experiment, the rest period was inserted before the first transition session, not after it. To conduct a study more similar to Ellson's, the rest period would need to be inserted after the first transition session (which is the counterpart to the first extinction session in Ellson's experiment). For this case, the model of J. A. Devenport and L. D. Devenport (1993) does predict more spontaneous recovery as the duration of the rest period increases, because the impact of the first transition session should decrease as this session is pushed farther back in time. To my knowledge, no such study has been conducted in a choice situation.

The indication that the difference between rest and no-rest conditions was larger in the first half of the experiment suggests that the passage of time may have less effect once subjects become accustomed to multiple rest days. (Sessions were usually conducted 6 days a week in Experiment 1, so it was only in Experiment 2 that the subjects were exposed repeatedly to 3 -day rest periods.) If further research shows that this is a reliable effect, it would be evidence against the theory of J. A. Devenport and L. D. Devenport (Equation 4), which predicts an unchanging, parameter-free inverse relation between time and the weight assigned to any past event.

\section{GENERAL DISCUSSION}

These two experiments, plus others that demonstrate spontaneous recovery in choice behavior (Mark \& Gallistel, 1993; Mazur, 1995), provide some clues about how past experiences are stored in an animal's memory, and how these experiences combine to affect present behavior. One of the simplest possible ways to represent past experiences in a choice situation would be to assign a single number to each alternative that represents the strength or value of that alternative (such as $V_{1}$ and $V_{2}$ in Equations 1-3) and then continually update that number as new choices are made. This strategy is incorporated, either explicitly or implicitly, in the many mathematical models that use exponentially weighted moving averages (e.g., Bush \& Mosteller, 1955; Couvillon \& Bitterman, 1985; Killeen, 1982; Staddon, 1988). This method of updating preferences over time is mathematically convenient, but, unfortunately, it cannot account for spontaneous recovery or other effects of the passage of time (e.g., J. A. Devenport \& L. D. Devenport, 1993; L. D. Devenport \& J. A. Devenport, 1994). If all of an animal's experiences with each choice alternative are reduced to a single number, there is no easy way to explain why choice responses often change dramatically from the end of one session to the beginning of the next. Even if we assumed that this number can change during the time between sessions, it would be difficult to explain why preference for the favored alternative sometimes becomes more extreme and sometimes less extreme from the end of one session to the beginning of the next. It would also be difficult to explain why such between-session changes are large when the reinforcement contingencies have changed recently but small when there has been no recent change.

In contrast, it is fairly easy to account for these between-session changes if we assume that an animal stores a separate representation for each choice alternative in every session and that the representations of the past several sessions are averaged at the start of each new session. As the computer simulations presented here have shown, this approach can predict both the direction and the relative size of the spontaneous recovery. If two alternatives have been equal for several sessions and then one suddenly becomes richer, spontaneous recovery in the direction of indifference will be observed (as in the transition sessions of Experiment 1). If one alternative has been richer for several sessions and then suddenly the two alternatives become equal, spontaneous recovery in the direction of increased preference will be observed (as in the recovery sessions of Experiment 1). Furthermore, Experiment 1 showed that the residual effects of a change in the reinforcement probabilities depend on both the magnitude of this change (i.e., whether the rich 
key delivered $70 \%$ or $90 \%$ of the reinforcers) and the number of sessions that this change was in effect. All of these findings are readily explained by assuming that choice behavior at the start of a new session reflects some type of average of the events of the past several sessions.

Although an averaging of past experiences can explain spontaneous recovery in choice behavior and related effects, the results obtained so far do not tell us much about the quantitative properties of this averaging process. The results do show that the events of the current session are given much more weight than events that occurred $24 \mathrm{~h}$ earlier (otherwise, there would not be such a rapid change in preference at the beginning of the 1 st transition day). The results of Experiment 2 suggest that the influence of past events may diminish over time, even when the subject receives no further experience with the choice alternatives during this time. The results of both experiments are consistent, at a qualitative level, with the theory of J. A. Devenport and L. D. Devenport (1993) that the weighting of past events diminishes according to an inverse function as time passes (Equation 4). However, the results do not discriminate between this equation and other possible weighted averages, and more subtle experimental manipulations will be needed to determine the quantitative properties of this weighting function.

Whereas the results suggest that an animal's memory contains separate representations of the response alternatives for each previous session, they do not rule out the possibility that more than one representation for each alternative may be stored in a single session. Some theories (e.g., Gibbon, Church, Fairhurst, \& Kacelnik, 1988; Heinemann, 1983) maintain that the animal stores a record of each trial or each reinforcer and that individual records can be retrieved at a later time. If we assume that the number or strengths of these individual records decrease over time, this type of theory can also account for spontaneous recovery in choice behavior. Thus, the evidence for spontaneous recovery does not set an upper limit on how many representations may be stored for each choice alternative, but it suggests that the number is greater than one, and probably at least one per session.

\section{REFERENCES}

Bailey, J. T., \& Mazur, J. E. (1990). Choice behavior in transition Development of preference for the higher probability of reinforcement. Journal of the Experimental Analysis of Behavior, 53, 409-422.

BUSH, R. R., \& MOSTELLER, F. (1955). Stochastic models for learning New York: Wiley.

Couvillon, P. A., \& Bitterman, M. E. (1985). Analysis of choice in honeybees. Animal Learning \& Behavior, 13, 246-252.

Couvillon, P. A., \& Bitterman, M. E. (1988). Compoundcomponent and conditional discrimination of colors and odors by honeybees: Further tests of a continuity model. Animal Learning \& Behavior, 16, 67-74.

Couvillon, P. A., \& Bitterman, M. E. (1991). How honeybees make choices. In L. J. Goodman \& R. C. Fisher (Eds.), The behaviour and physiology of bees (pp. 116-130). Wallingford, U.K.: CAB International.

Devenport, J. A., \& Devenport, L. D. (1993). Time-dependent decisions in dogs (Canis familiaris). Journal of Comparative Psychology, 107, 169-173.

DevenPort, L. D., \& Devenport, J. A. (1994). Time-dependent averaging of foraging information in least chipmunks and goldenmantled ground squirrels. Animal Behaviour, 47, 787-802.

DREYFUS, L. R. (1991). Local shifts in relative reinforcement rate and time allocation on concurrent schedules. Journal of Experimental Psychology: Animal Behavior Processes, 17, 486-502.

ELLSON, D. G. (1938). Quantitative studies of the interacton of simple habits: 1. Recovery from specific and generalized effects of extinction. Journal of Experimental Psychology, 23, 339-358.

EsTES, W. K. (1955). Statistical theory of spontaneous recovery and regression. Psychological Review, 62, 145-154.

Gibbon, J., ChurCh, R. M., Fairhurst, S., \& Kacelnik, A. (1988). Scalar expectancy theory and choice between delayed rewards. Psychological Review, 95, 102-114.

HeinemanN, E. G. (1983). A memory model for decision processes in pigeons. In M. L. Commons, R. J. Herrnstein, \& A. R. Wagner (Eds.), Quantitative analyses of behavior: IV. Discrimination processes (pp. 1-19). Cambridge, MA: Ballinger.

KACELNIK, A., KREBS, J. R., \& ENS, B. (1987). Foraging in a changing environment: An experiment with starlings (Sturnus vulgaris). In M. L. Commons, A. Kacelnik, \& S. J. Shettleworth (Eds.), Quantitative analyses of behavior: Vol. 6. Foraging (pp. 63-87). Hillsdale, NJ: Erlbaum.

KILLEEN, P. (1982). Incentive theory. In D. J. Bernstein (Ed.), Nebraska Symposium on Motivation, 1981: Response structure and organization (pp. 169-216). Lincoln: University of Nebraska Press.

Mark, T. A., \& Gallistel, C. R. (1993, November). The microstructure of matching. Paper presented at the meeting of the Psychonomic Society, Washington, DC.

MARK, T. A., \& Gallistel, C. R. (1994). Kinetics of matching. Journal of Experimental Psychology: Animal Behavior Processes, 20, 79-95.

MAZUR, J. E. (1992). Choice behavior in transition: Development of preference with ratio and interval schedules. Journal of Experimental Psychology: Animal Behavior Processes, 18, 364-378.

MAZUR, J. E. (1995). Development of preference and spontaneous recovery in choice behavior with concurrent variable-interval schedules. Animal Learning \& Behavior, 23, 93-103.

MAZUR, J. E., \& RATTI, T. A. (1991). Choice behavior in transition: Development of preference in a free-operant procedure. Animal Learning \& Behavior, 19, 241-248.

Myerson, J., \& HALE, S. (1988). Choice in transition: A comparison of melioration and the kinetic model. Journal of the Experimental Analysis of Behavior, 49, 291-302.

Myerson, J., \& Miezin, F. M. (1980). The kinetics of choice: An operant systems analysis. Psychological Review, 87, 160-174.

SPEAR, N. E. (1967). Retention of reinforcer magnitude. Psychological Review, 74, 216-234.

SPEAR, N. E. (1971). Forgetting as retrieval failure. In W. K. Honig \& P. H. R. James (Eds.), Animal memory (pp. 45-109). New York: Academic Press.

Staddon, J. E. R. (1988). Quasi-dynamic choice models: Melioration and ratio invariance. Journal of the Experimental Analysis of Behavior, 49, 303-320.

StAdDON, J. E. R., \& HoRNER, J. M. (1989). Stochastic choice models: A comparison between Bush-Mosteller and a source-independent reward-following model. Journal of the Experimental Analysis of Behavior, 52, 57-64.

(Manuscript received August 23, 1994; revision accepted for publication January 8,1995 .) 